\title{
Evaluación de los conocimientos y habilidades de los alumnos del grado de Farmacia: Prueba ECOE (Evaluación Clínica Objetiva Estructurada)
}

\author{
María Araceli Calatayud-Pascual', Cristina Balaguer-Fernández', Luis Salar-Ibáñez ${ }^{1,2}$, Lucrecia Moreno-Royo', \\ Alicia López-Castellano ${ }^{1}$ \\ 1. Departamento de Farmacia. Facultad Ciencias de la Salud. Universidad CEU Cardenal Herrera, Valencia, España. 2. Farmacéutico comunitario
}

\section{PALABRAS CLAVE}

Habilidades clínicas, competencias, ECOE, estudiantes de farmacia, simulación

\section{ABREVIATURAS}

ECOE: evaluación clínica objetiva estructurada

UCH-CEU: Universidad Cardenal Herrera - CEU

\section{KEYWORDS}

Clinical skills, competencies, OSCE (objective structured clinical examination), pharmacy students, simulation

\section{RESUMEN}

La enseñanza universitaria evoluciona hacia la formación basada en problemas. La prueba ECOE (Evaluación Clínica Objetiva Estructurada) permite evaluar la capacidad del alumno en tres de los cuatro escalones de la pirámide de Miller; saber, saber cómo y demostrar cómo (conocimientos, habilidades y actitudes). Esta es la prueba de evaluación de las practicas tuteladas y en la Universidad Cardenal Herrera CEU se ha impartido en los dos últimos cursos a un total de 79 alumnos con resultados satisfactorios. El alumno debe pasar por 5 estaciones donde en 5 minutos en cada una debe resolver el problema práctico que se le plantea. Un profesor entrenado hace de paciente y otro profesor evalúa las habilidades del alumno.

Luego se pasó una encuesta de satisfacción anónima a los alumnos siendo el resultado de $4,12 / 6$.

Es necesario ir modificando los problemas planteados a los alumnos para que cada vez más se adapten a la realidad que se encontraran al obtener el grado como profesionales farmacéuticos.

Assessment of the knowledge and skills of Pharmacy graduates: Objective Structured Clinical Examination (OSCE)

\section{ABSTRACT}

Higher education evolves towards Problem Based Learning. The Objective Structured Clinical Examination (OSCE) assesses students' competencies in three of the four levels of Miller's pyramid; Knows, Knows How, and Shows How (knowledge, competence, and performance). In the last two years, a total of 79 students at Cardenal Herrera University CEU were assessed with this examination, with good results. Student are asked to go through 5 stations, in which they have 5 minutes to resolve a practical problem. A trained teacher acts as the patient, while another evaluates the student's abilities.

Next, an anonymous satisfaction survey is given to the students, with the result $4,12 / 6$.

The problems presented to the students must be changed constantly so that each time they increasingly adapt to the situations they will find in their real-life practice once the graduate as professional pharmacists.
Financiación: ninguna.

Conflicto de intereses: ninguno.

Cite este artículo como: Calatayud-Pascual MA, Balaguer-Fernández C, Salar-lbáñez L, Moreno-Royo L, López-CasteIlano A. Evaluación de los conocimientos y habilidades de los alumnos del grado de Farmacia: Prueba ECOE (Evaluación Clínica Objetiva Estructurada). Farmacéuticos Comunitarios. 2020 Mar 06; 12(1):29-34. doi:10.5672/FC.2173-9218.(2020/ Vol12).001.06

Correspondencia: Maria Araceli Calatayud-Pascual (maria.calatayud@uchceu.es).

ISSN 1885-8619 @SEFAC (Sociedad Española de Farmacia Familiar y Comunitaria). Todos los derechos reservados. 
Una prueba ECOE es una Evaluación Clínica Objetiva Estructurada y se define como "un método de evaluación de competencias clínicas en el que los componentes de estas competencias se evalúan de forma planificada o estructurada, prestando especial interés a la objetividad del examen" (1).

La potencia de este formato de prueba se sustenta en la mezcla de métodos de evaluación, de manera que es capaz de explorar suficientemente tres de los cuatro niveles de la pirámide de Miller: conocimientos, habilidades y actitudes (véase figura 1).

La prueba ECOE consiste en el desarrollo de sucesivas pruebas, denominadas "estaciones", en un circuito que simula situaciones reales (3). En cada estación, en función de un tiempo determinado, los estudiantes evaluados interactúan con un paciente simulado para demostrar determinadas habilidades. Los pacientes simulados son personas entrenadas (profesores, investigadores y colaboradores de la universidad) que representan problemas que pueden presentar los pacientes de manera real. En cada una de las estaciones, un observador anteriormente entrenado asigna una puntación de acuerdo con una escala previamente establecida. Los estudiantes, al tener la certeza de que sus habilidades y métodos de comunicación van a ser evaluados, muestran un mayor interés por aprenderlos, incluso reclaman de sus profesores de prácticas más tutorías. Los profesores, viendo el interés mostrado, se sienten también motivados, por lo que el resultado final es una mejora general del proceso de enseñanza/aprendizaje.

En los últimos años, la educación farmacéutica ha evolucionado considerablemente hacia una profesión más orientada al paciente, presentando mayor énfasis la enseñanza basada en problemas. En este sentido, la ECOE, debido a su fiabilidad y validez, se ha convertido en la prueba estándar para la evaluación de las habilidades clínicas de los estudiantes universitarios de farmacia de todo el mundo (4).

En la actualidad, la ECOE se utiliza como metodología docente para evaluar competencias profesionales en Farmacia en el Reino Unido, Estados Unidos (EE.UU.) y Canadá (5), suponiendo un modelo perfecto de examen para evaluar las competencias y habilidades desarrolladas por los estudiantes del grado en Farmacia en estos países $(6,7,8)$.

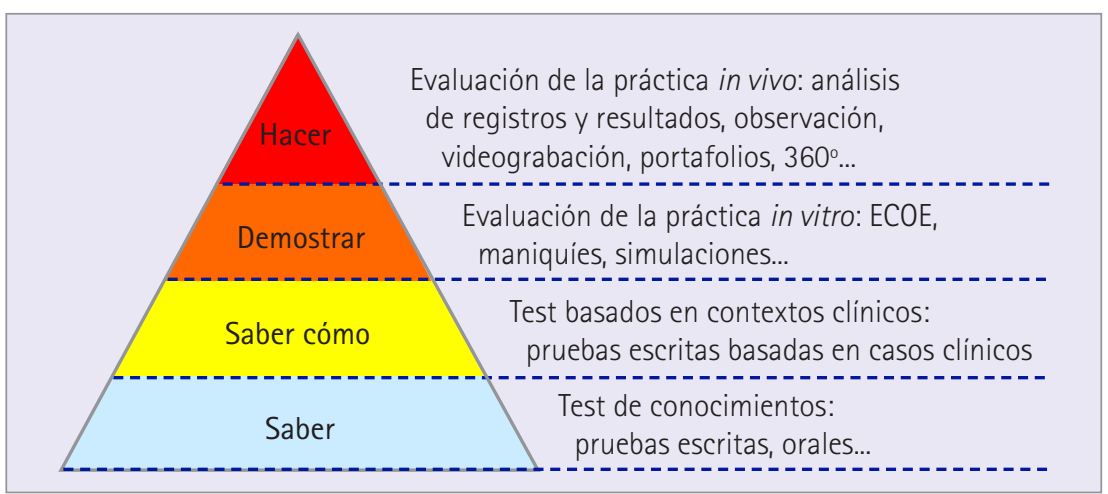

Figura 1 Relación entre el nivel de competencia según la pirámide de Miller y los instrumentos susceptibles de ser utilizados. Adaptado de la referencia (2)

La Real Sociedad Farmacéutica de Gran Bretaña, junto con los métodos tradicionales de evaluación, defiende la inclusión del aprendizaje basado en competencias y la evaluación mediante la prueba ECOE (9). Por otra parte, en EE.UU., el incremento de comunicaciones de investigación presentadas en los foros académicos de la titulación de Farmacia demuestra el creciente interés de esta técnica de evaluación $(10,11)$.

En nuestra universidad, el examen ECOE se aplica desde el curso académico 2015-16 en la titulación de Medicina. Debido al éxito generado y a partir de un Proyecto CEU Innova con profesores de Farmacia, hace dos cursos académicos se implantó también esta prueba práctica en la asignatura Prácticas Tuteladas de la titulación de Farmacia que se imparte en la sede de Valencia de la Universidad CEU Cardenal Herrera (UCH-CEU) (12).

Para ello, se establecieron una serie de acciones:

1. Revisión bibliográfica y análisis de los planes de estudios de Farmacia de otras Facultades donde evalúan con esta prueba ECOE. Para ello se determinaron:

a) Especificaciones de cada situación (prueba práctica o estación) a la cual el alumno se va a enfrentar: número de la estación, área de conocimiento, ámbito y evaluación. b) Estaciones. Tipo de estación: práctica mediante actores, examen en papel, etc.

c) Personal. Personas responsables de su ejecución. Actores necesarios para completar la actividad práctica de la forma más real posible. Número de profesores y tutores necesarios en cada estación (actor, evaluador).

d) Materiales. Recursos necesarios para cada una de las pruebas. Material de laboratorio, fuentes de información, medicamentos, productos sanitarios y cosméticos, etc.

e) Prueba. Duración de cada estación y tipo de prueba a desarrollar.

f) Evaluación de resultados. Elaboración de rúbricas estandarizadas (checklist) y consensuadas entre los tutores y profesores a fin de asegurar la objetividad, sencillez y claridad de la prueba.

2. Realización de diversas sesiones informativa, tanto al profesorado como al alumnado, sobre la ECOE de Farmacia.

3. Obtención del Mapa de Competencias. Se identificaron los contenidos de la asignatura para poder establecer según las competencias de Prácticas Tuteladas del grado de Farmacia las competencias adecuadas para la prueba ECOE. De esta forma, se diseñaron casos concretos acordes a las competencias acotadas en el Mapa de Competencias (véase tabla 1). Dicho mapa fue explicado a cada uno de los profesores.

Tabla 1 Competencias establecidas para la prueba ECOE del grado de Farmacia

Componentes competenciales Porcentaje prueba ECOE

\begin{tabular}{|l|c|}
\hline Manejo terapéutico del paciente & 30 \\
\hline Laboratorio de formulación y análisis & 25 \\
\hline Prevención y promoción de la salud & 15 \\
\hline Gestión y planificación farmacéutica & 10 \\
\hline Habilidades de comunicación con el paciente & 10 \\
\hline Relaciones interprofesionales & 5 \\
\hline Fuentes de información del medicamento & 5 \\
\hline TOTAL & 100 \\
\hline
\end{tabular}




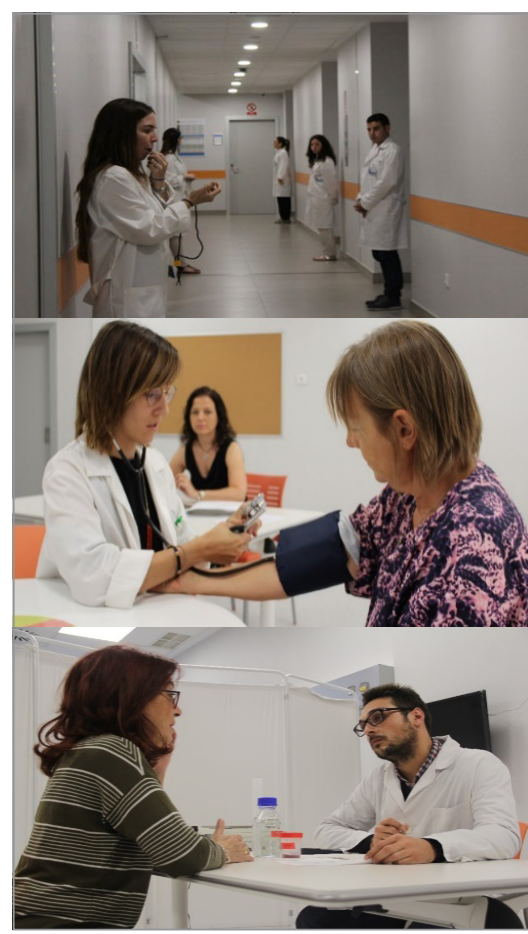

Figura 2 Imágenes obtenidas durante las pruebas ECOE realizadas

4. Elaboración de una ficha genérica de los casos-estaciones, a fin de que cada profesor pudiera diseñar diferentes casos-estaciones para su posterior puesta a punto. Se preparó un formulario de obligada cumplimentación por parte del profesorado.

En los dos últimos cursos académicos, las pruebas ECOE realizadas en la titulación de Farmacia de la UCHCEU se han realizado en el Centro de Simulación Avanzada de la Facultad de Salud de dicha Universidad (véase figura 2).

Las pruebas han consistido en un circuito multidisciplinar de cinco estaciones (véase ejemplo en la figura 3). El tiempo de duración de cada estación ha sido de 5 minutos y la duración máxima de la prueba, 30 minutos. Durante ese tiempo, 5 minutos, el alumno lee el caso y entra a la estación para resolverlo. En el anexo 1 se describe una de las estaciones diseñadas.

En dichas pruebas han participado un total de 79 alumnos de $5^{\circ}$ curso matriculados en la asignatura Practicas Tuteladas. En el primer curso en el que tuvo lugar la prueba, la puntuación media obtenida por los 30 alumnos evaluados fue de 6,2/10. En el segundo curso, la puntación media obtenida por los 49 alumnos

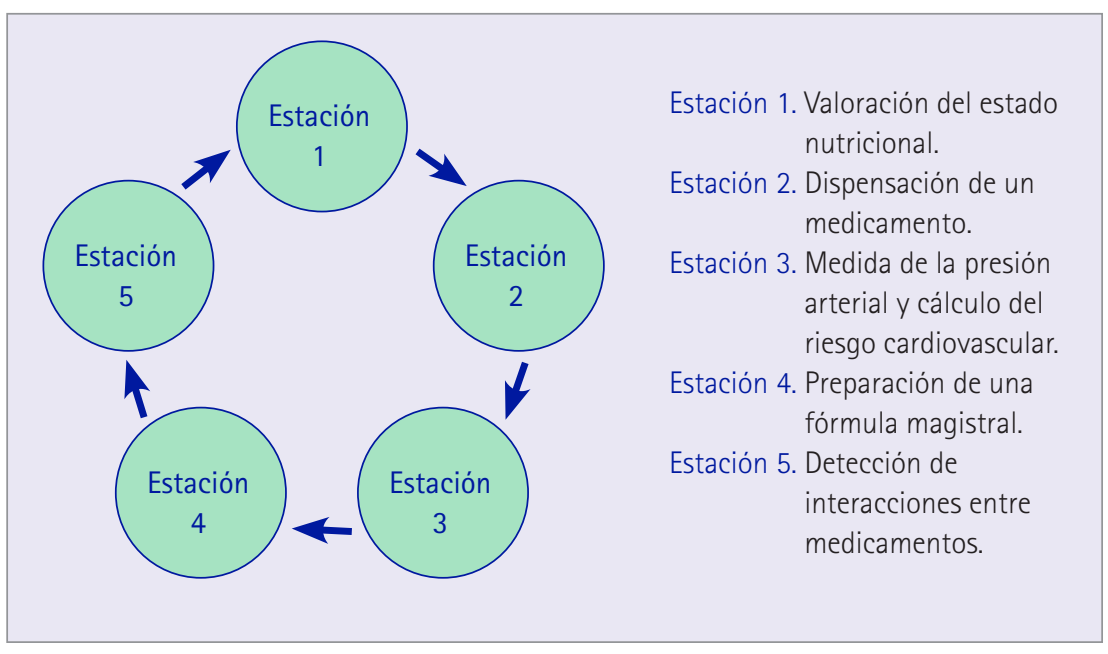

Figura 3 Ejemplo de uno de los circuitos de estaciones prueba ECOE evaluados fue de 7,0/10, algo superior a la obtenida el curso anterior. No obstante, los resultados de cada estación sugieren aspectos que se pueden mejorar. Así, es deseable que algunas áreas de conocimiento propias de la titulación tengan una mayor participación en la formulación de preguntas y casos para dicha prueba.

Los datos obtenidos a partir de la encuesta de satisfacción realizada a los estudiantes (véase tabla 2) muestran la satisfacción del alumnado con la prueba desarrollada. El valor medio obtenido fue de 4,95/6, siendo el mínimo valor alcanzado en los ítems de 4,12/6.

Por lo expuesto con anterioridad, se considera que la prueba ECOE es una herramienta muy útil para la evaluación de competencias de alumnos de grado en Farmacia. Se trata de una prueba de formato flexible, que permite conocer si los estudiantes y, en consecuencia, los programas docentes, cumplen el objetivo de "formar en competencias" de acuerdo con las directrices del plan Bolonia.
Tabla 2 Encuesta de satisfacción con la prueba ECOE realizada por el alumnado

\begin{tabular}{|l|l|l|l|l|l|l|l|}
\hline 1 & La prueba ECOE ha sido organizada correctamente & 1 & 2 & 3 & 4 & 5 & 6 \\
\hline 2 & $\begin{array}{l}\text { La información presentada para la prueba ECOE (fecha, } \\
\text { hora, lugar y objetivos) ha sido detallada con tiempo } \\
\text { suficiente para su conocimiento }\end{array}$ & 1 & 2 & 3 & 4 & 5 & 6 \\
\hline 3 & $\begin{array}{l}\text { En general, icómo le ha parecido la organización de las } \\
\text { diferentes estaciones de la ECOE? }\end{array}$ & 1 & 2 & 3 & 4 & 5 & 6 \\
\hline 4 & $\begin{array}{l}\text { Las caracteristicas del centro de prácticas alcanzan sus } \\
\text { expectativas para la realización de esta prueba }\end{array}$ & 1 & 2 & 3 & 4 & 5 & 6 \\
\hline 5 & $\begin{array}{l}\text { El material empleado en cada estación ha sido suficiente } \\
\text { para lograr los objetivos esperados }\end{array}$ & 1 & 2 & 3 & 4 & 5 & 6 \\
\hline 6 & $\begin{array}{l}\text { La duración de la prueba ECOE, en su totalidad, es } \\
\text { factible y aceptable para usted }\end{array}$ & 1 & 2 & 3 & 4 & 5 & 6 \\
\hline 7 & $\begin{array}{l}\text { El tiempo asignado para realizar las diferentes estaciones } \\
\text { ha sido suficiente }\end{array}$ & 1 & 2 & 3 & 4 & 5 & 6 \\
\hline 8 & $\begin{array}{l}\text { Los evaluadores yactores de cada estación han adoptado } \\
\text { conmigo una actitud de trato respetuoso, humano y } \\
\text { profesional }\end{array}$ & 1 & 2 & 3 & 4 & 5 & 6 \\
\hline 9 & $\begin{array}{l}\text { La ECOE aporta crecimiento personal y/o profesional y } \\
\text { considero que este aprendizaje será útil para mí en el } \\
\text { futuro }\end{array}$ & 1 & 2 & 3 & 4 & 5 & 6 \\
\hline 10 & $\begin{array}{l}\text { En general, estoy satisfecho con la actuación que he } \\
\text { realizado en la ECOE }\end{array}$ & 1 & 2 & 3 & 4 & 5 & 6 \\
\hline
\end{tabular}

(1) Completamente en desacuerdo. (2) Muy en desacuerdo. (3) En desacuerdo. (4) De acuerdo. (5) Muy de acuerdo. (6) Completamente de acuerdo. 


\section{Referencias bibliográficas}

1. Harden RM. What is an OSCE? Med Teach. 1988; 10(1):19-22. doi:10.3109/ 01421598809019321

2. Miller G.E. The assessment of clinical skills/competence/performance. Acad Med. 1990; 65 (suppl 9): 63-67. doi: 10.1097/00001888-199009000-00045

3. Kamran Z. Khan, Sankaranarayanan Ramachandran, Kathryn Gaunt $\&$ Piyush Pushkar. The Objective Structured Clinical Examination (OSCE): AMEE Guide No. 81. Part I: An historical and theoretical perspective, Medical Teacher. 2013: 35:9, e1437-e1446. doi:10.3109/0142159X.2013.818634

4. Shirwaikar A. Objective structured clinical examination (OSCE) in pharmacy education a trend. Pharm Pract. 2015; 13(4): 627. doi:10.18549/ PharmPract.2015.04.627

5. The Pharmacy Examining Board of Canada [Sede Web] Toronto, Canada.
15/01/2020 [último acceso] https:// www.pebc.ca/index.php/ci_id/3374/ la_id/1.htm

6. Hal Sosabowski M, Gard P.R. Pharmacy Education in the United Kingdom. Am J Pharm Educ. 2008 Dec 15; 72(6): 130. doi:10.5688/aj7206130

7. Sturpe DA. Objective structured clinical examinations in doctor of pharmacy programs in the United States. Am J Pharm Educ. 2010; 74(8):148. doi:10.5688/aj7408148

8. Austin Z, O’Byrne CC, Pugsley J, Munoz LQ. Development and validation processes for an objective structured clinical examination (OSCE) for entry-to-practice certification in pharmacy: the Canadian experience. Am J Pharm Educ. 2003; 67(3):76. doi:10. 5688/aj670376

9. Brian Kirton S, Kravitz L. Objective Structured Clinical Examinations (OSCEs) Compared With Traditional
Assessment Methods. Am J Pharm Educ. 2011; 10; 75(6): 111. doi:10.5688/ ajpe756111

10. Young A. 107th Annual Meeting of the American Association of Colleges of Pharmacy. San Diego, CA. 2006. Making learning portable: continuing education (CE).

11. Stowe CD, O'Brien CE, Warmack TS, Gardner SF. 108th Annual Meeting of the American Association of Colleges of Pharmacy. Orlando, FL. 2007. Communication skill development: OSCE assessment of lay and healthcare provider encounters.

12. Balaguer-Fernández C, Moreno L, Calatayud-Pascual M.A., López-Castellano A. An Objective Structured Clinical Examination (OSCE) to evaluate clinical/communication skills of pharmacy students. IPAP18 Innovations in Pharmacy. Abstract book; p. 287. 


\section{Anexo 1. Diseño de estación ECOE de dispensación}

\section{Situación}

Cuando el alumno entra en la sala se encuentra con una mesa a modo de mostrador, con 7 medicamentos encima, y al profesor que hace de paciente. El profesor entrega al alumno una hoja que contiene un modelo de receta electrónica y le dice: "Por favor, ¿puede darme esto?". El alumno debe entonces proceder a la dispensación.

Se indicará al alumno que las líneas en rojo no están disponibles para ese dia (en la receta sale todo en negro, pero en la pantalla del ordenador aparece en rojo las líneas que no están disponibles).

En las recetas originales los medicamentos nuevos aparecen en negrita y así están en el modelo adjunto. Esto debe saberlo el alumno.

\section{Información que tiene el profesor para responder a las posibles preguntas que pueda hacer el alumno}

El paciente es un hombre de 62 años, fumador, que acude al médico de primaria (MFC) por un resfriado y éste le receta salbutamol $100 \mu \mathrm{g}$ inhalado y cefuroxima.

Hace 9 años tuvo un evento cardiovascular y desde entonces está en tratamiento con acenocumarol, furosemida y atorvastatina. Eso le provocó insomnio y desde entonces toma lorazepam por la noche para dormir.

Hace tres años le diagnosticaron diabetes II y desde entonces toma metformina.

En la receta de hoy tiene todo disponible para retirar excepto atorvastatina y metformina.

El alumno debe coger los 5 medicamentos disponibles (dispone de los 7 medicamentos que hay en la receta) y presentárselos al paciente. Este, entonces, dirá que acenocumarol y furosemida no los quiere.

\section{Intervención del alumno}

1. En algún momento, el alumno debe preguntar para quién son los medicamentos.

2. Debe preguntar por qué no quiere los que no se lleva, y la respuesta será "porque tengo". El alumno debe investigar el cumplimiento.

2a. En el caso del acenocumarol el paciente dirá que la pauta es variable y siempre tiene más de lo necesario. Es correcto, el alumno no debe hacer nada.

2b. En el caso de la furosemida (40 mg) dirá que no se la toma porque le hace orinar demasiado y no puede salir de casa. El alumno debería insistir en la importancia de tomarlo y proponer cambiar la hora de administración si eso mejora la aceptación.

3. Benzodiacepina. Debe preguntar "desde cuándo lo toma" y recomendar comentar con el médico para su deshabituación.

4. Con los medicamentos nuevos debe comprobar si el paciente conoce su indicación, posología, pauta, forma de uso y duración del tratamiento y, además:

4a. Salbutamol (100 $\mu \mathrm{g}$ ). Informar de su uso. Manejo de inhaladores (sería suficiente con que empezara a informar, probablemente no habrá tiempo de hacerlo con detalle).

4b. Cefuroxima. Informar de que es un antibiótico e insistir en terminar el tratamiento.

5. Debe preguntar si fuma y recomendar el abandono del tabaco.

\section{Valoración}

En una dispensación pueden surgir otras acciones. En la presente, las más importantes y obvias son las planteadas y son las que se evaluarán en la estación.

\begin{tabular}{|c|c|}
\hline Pregunta & Puntuación \\
\hline 1 & 2 \\
\hline 2 & 1 \\
\hline $2 a$ & 1 \\
\hline $2 b$ & 1 \\
\hline 3 & 1 \\
\hline 4 & 1 \\
\hline $4 a$ & 1 \\
\hline $4 b$ & 1 \\
\hline 5 & 1 \\
\hline
\end{tabular}




\section{Receta electrónica}

Paciente: hombre de 62 años

Fecha 04/07/2019

\begin{tabular}{|l|c|c|c|}
\hline Medicamento & Fecha inicio & Fecha fin & Médico \\
\hline $\begin{array}{l}\text { Orfidal } 1 \text { mg } 50 \text { comprimidos. } 1 \text { comprimido cada } 24 \text { horas durante } 257 \text { días. } \\
\text { Principio activo - Lorazepam }\end{array}$ & $14 / 01 / 2010$ & $10 / 10 / 2019$ & PSI \\
\hline $\begin{array}{l}\text { Sintrom } 1 \text { mg } 60 \text { comprimidos. } 3 \text { comprimidos cada } 24 \text { horas. CRÓNICO. } \\
\text { Principio activo - Acenocumarol }\end{array}$ & $14 / 01 / 2010$ & $27 / 03 / 2020$ & CAR \\
\hline $\begin{array}{l}\text { Seguril } 40 \text { mg } 30 \text { comprimidos. } 1 \text { comprimido cada } 24 \text { horas. CRÓNICO. } \\
\text { Tomar preferentemente por la mañana. } \\
\text { Principio activo - Furosemida }\end{array}$ & $\mathbf{1 4 / 0 1 / 2 0 1 0}$ & $\mathbf{2 7 / 0 3 / 2 0 2 0}$ & CAR \\
\hline $\begin{array}{l}\text { Atorvastatina } 40 \text { mg } 28 \text { comprimidos. } 1 \text { comprimido cada } 24 \text { horas. CRÓNICO. } \\
\text { Tomar preferentemente por la noche }\end{array}$ & $\mathbf{1 4 / 0 1 / 2 0 1 0}$ & $\mathbf{1 2 / 1 2 / 2 0 1 9}$ & MFC \\
\hline $\begin{array}{l}\text { Dianben } 850 \text { mg. } 50 \text { comprimidos. } 1 \text { comprimido cada } 12 \text { horas durante } 198 \text { dias. } \\
\text { Principio activo - Metformina }\end{array}$ & $\mathbf{2 0 / 0 6 / 2 0 1 7}$ & $\mathbf{1 0 / 1 1 / 2 0 1 9}$ & MFC \\
\hline $\begin{array}{l}\text { Ventolin inhalador. Una pulsación cada } \mathbf{1 2} \text { horas durante } \mathbf{3 0} \text { días. } \\
\text { Principio activo - Salbutamol }\end{array}$ & $\mathbf{0 4 / 0 7 / 2 0 1 9}$ & $\mathbf{0 3 / 0 8 / 2 0 1 9}$ & MFC \\
\hline $\begin{array}{l}\text { Cefuroxima } \mathbf{5 0 0} \text { mg } \mathbf{2 0} \text { comprimidos. } \mathbf{1} \text { comprimido cada } \mathbf{1 2} \text { horas durante } \\
\mathbf{1 0} \text { días }\end{array}$ & $\mathbf{0 4 / 0 7 / 2 0 1 9}$ & $\mathbf{1 4 / 0 7 / 2 0 1 9}$ & MFC \\
\hline
\end{tabular}

